\title{
手性钴(III)阴离子控制的铜催化环状芳基高碘盐的开环反应
}

\author{
段伟良*
}

(扬州大学化学化工学院 江苏扬州 225002)

\section{Copper-Catalyzed Ring Opening of Cyclic Diaryliodonium Salts Controlled by Chiral Cobalt(III) Anion}

\author{
Duan, Weiliang* \\ (College of Chemistry and Chemical Engineering, Yangzhou University, Yangzhou, Jiangsu 225002)
}

轴手性(axial chirality)是由于存在相邻较大体积的 基团的位阻排斥作用，导致芳基-芳基单键不能自由旋 转而产生的现象 ${ }^{[1]}$. 轴手性化合物广泛存在于手性配体 和催化剂、天然产物、药物分子(图 1)中, 这类化合物的 催化不对称合成方法一直是研究者的关注热点. 已知报 道的催化合成路线主要包括金属参与的芳基之间的<smiles>Oc1ccc2ccccc2c1-c1c(O)ccc2ccccc12</smiles>

$(R)$-Binol<smiles>Pc1ccccc1-c1ccc2ccccc2c1-c1c(-c2ccccc2)ccc2ccccc12</smiles>

$(R)$-Binap

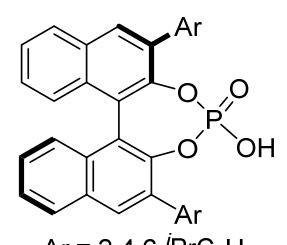

$\mathrm{Ar}=2,4,6-\mathrm{PrC}_{6} \mathrm{H}_{2}$ $(R)$-TRIP

图 1 轴手性化合物范例

Figure 1 Representative axially chiral compounds

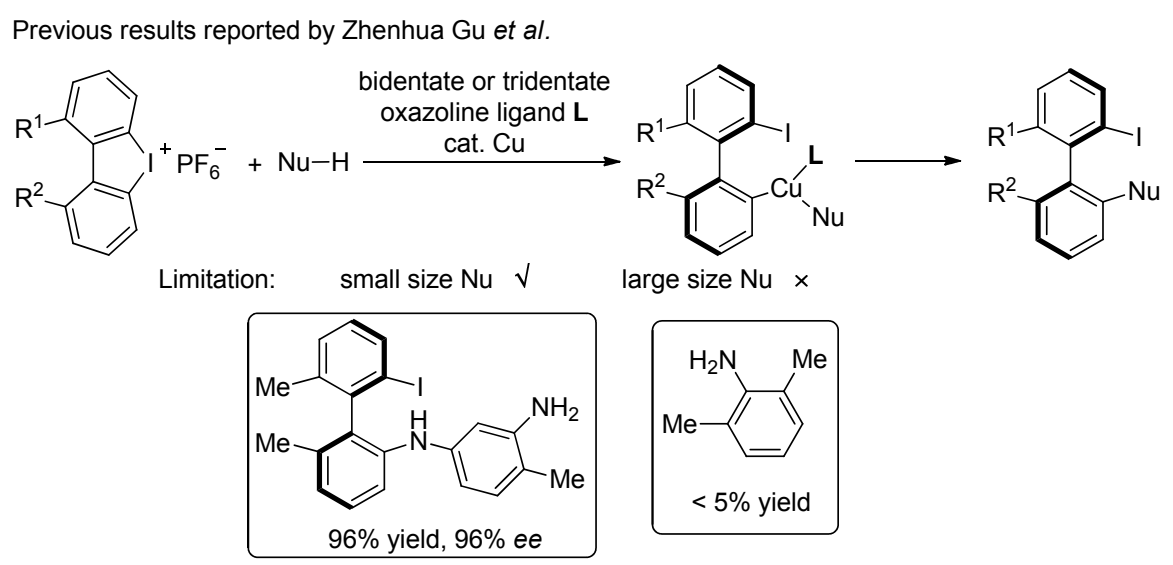

图式 1 使用含氮配体的实验结果

Scheme 1 Experimental results with $\mathrm{Cu} /$ nitrogen ligand

* Corresponding author. E-mail: duanwl@yzu.edu.cn. Published online December 10, 2020. 
过渡金属催化的不对称反应中使用的手性配体和 金属, 通常通过配位键结合形成相对稳定的活性催化剂 来控制反应的立体选择性. 在金属催化中, 使用手性阴 离子配体比如手性磷酸等来控制反应的立体选择性, 是 近年来兴起的一种有效途径, 在各种催化过程中取得了 优异的结果 ${ }^{[5]}$. 然而手性阴离子催化剂的类型还不够多 样化，所适用的金属催化反应类型仍需进一步拓展.

针对环状二芳基高碘盐和大位阻芳胺的对映选择 性开环反应不能有效进行这一问题, 中国科学技术大学
化学系顾振华与安徽农业大学应用化学系俞杰合作报 道了使用催化量的手性三价钴阴离子 [6]，可以在大位阻 芳胺与高碘盐的开环反应中实现高立体选择性(up to $98 \% e e)$ 控制 ${ }^{[7]}$, 而使用 $(R)$-联萗酚衍生的手性磷酸仅获 得 $6 \%$ ee (Scheme 2).

作者在最优条件下对底物范围做了一定拓展，发现 反应可以容忍多种供电子和吸电子取代基, 杂环芳胺含 有噻吩基团也被用于反应之中，得到各种高立体选择性 的轴手性化合物(Scheme 3). 产物的碘取代基可通过偶

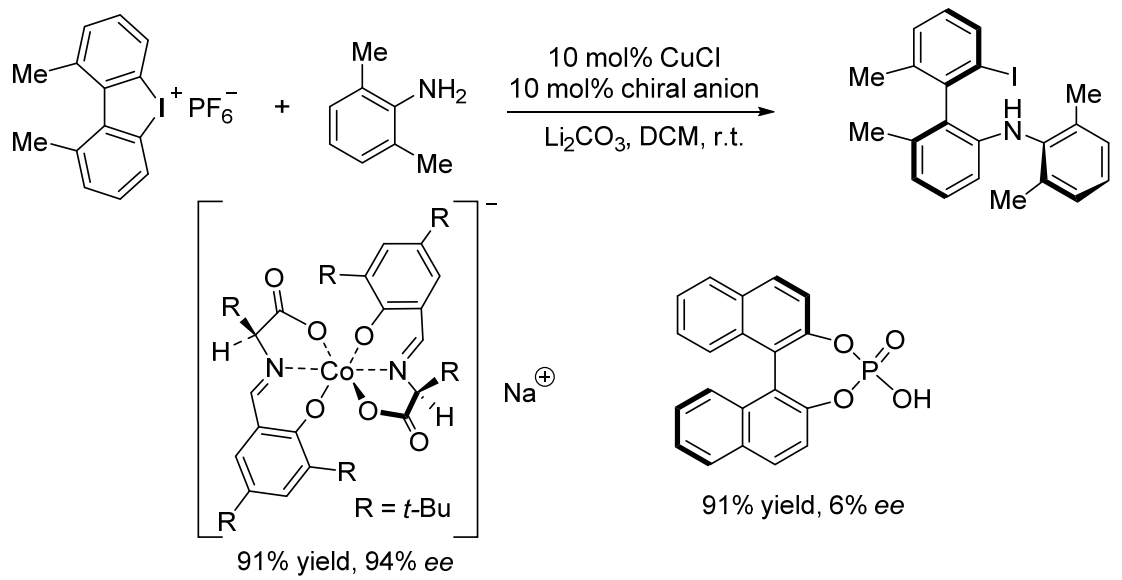

图式 2 铜催化环状高碘盐不对称开环合成轴手性化合物

Scheme 2 Cu-Catalyzed asymmetric ring opening of cyclic diaryliodoniun salts for the synthesis of axially chiral compounds

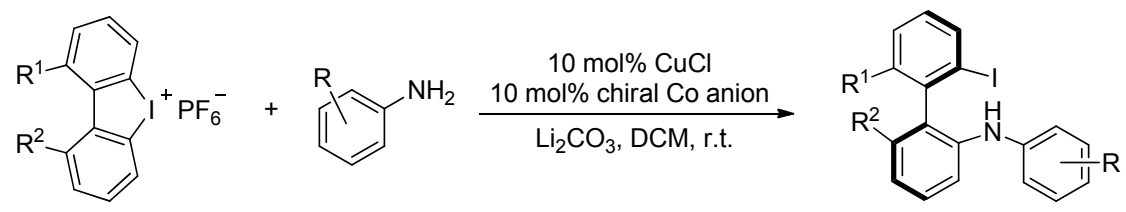<smiles>CCc1cccc(CC)c1Nc1cccc(C)c1-c1c(C)cccc1I</smiles>

$83 \%$ yield, $96 \%$ ee<smiles>CC(=O)c1cccc(C)c1Nc1cccc(C)c1-c1c(C)cccc1C</smiles>

$99 \%$ yield, $81 \%$ ee

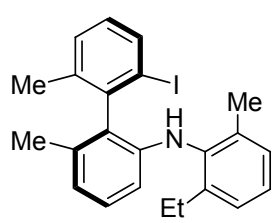

$79 \%$ yield, $95 \%$ ee<smiles>Cc1cccc(C)c1-c1c(C)cccc1Nc1c(C)ccc2ccccc12</smiles>

$56 \%$ yield, $82 \%$ ee

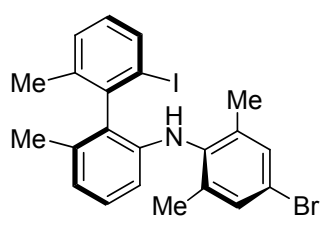

$81 \%$ yield, $93 \%$ ee

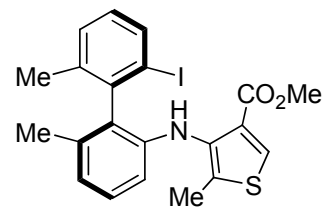

$88 \%$ yield, $87 \%$ ee<smiles>CCCCOc1cc(C)c(Nc2cccc(C)c2-c2c(C)cccc2C)c(C)c1</smiles><smiles>Cc1ccc(I)c(-c2c(Nc3c(C)cccc3C)ccc(C)c2C)c1C</smiles>

$94 \%$ yield, $95 \%$ ee

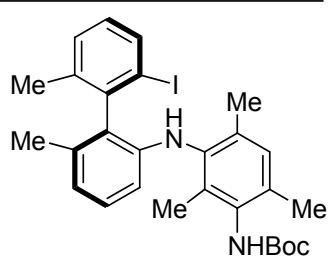

$67 \%$ yield, $91 \%$ ee<smiles>Cc1ccc(I)c(-c2c(Nc3ccccc3Br)ccc(C)c2C)c1C</smiles>

$65 \%$ yield, $71 \%$ ee<smiles>Cc1cccc(Nc2cccc(Cl)c2-c2c(Cl)cccc2I)c1C</smiles>

$64 \%$ yield, $95 \%$ ee

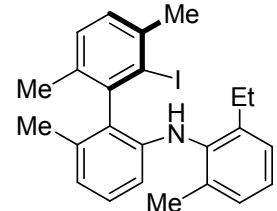

$86 \%$ yield, $98 \%$ ee<smiles>Cc1cccc(C)c1Nc1ccc2ccccc2c1-c1c(I)ccc2ccccc12</smiles>

$78 \%$ yield, $95 \%$ ee

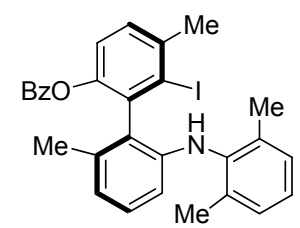

$84 \%$ yield, $93 \%$ ee

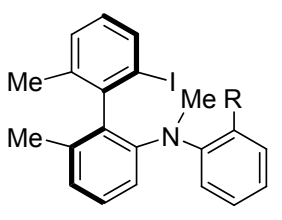

$\mathrm{R}=\mathrm{H}, 17 \%$ yield, $77 \%$ ee $\mathrm{R}=\mathrm{Me}$, no reaction

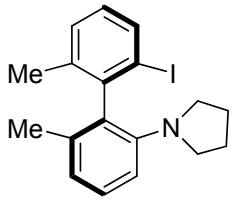

$48 \%$ yield, $51 \%$ ee

图式 3 底物范围

Scheme 3 Substrate scope 
联反应, 硅氢还原转化为轴手性磷氮配体. 在随后的氢 谱核磁实验研究表明, 加入手性钴阴离子后, 高碘盐芳 基上的氢的化学位移有明显的变化, 表明有紧密离子对 的形成. 对比实验显示使用 $\mathrm{NaPF}_{6} 、 \mathrm{NaOTf} 、 \mathrm{NaB}\left(\mathrm{Ar}^{\mathrm{F}}\right)_{4}$ 为添加剂时, 反应转化率仅为 $20 \% \sim 30 \%$, 这些结果说 明手性钴阴离子在此反应中有显著促进作用. 作者也提 出了可能的催化过程(Scheme 4). 手性钴阴离子可以与

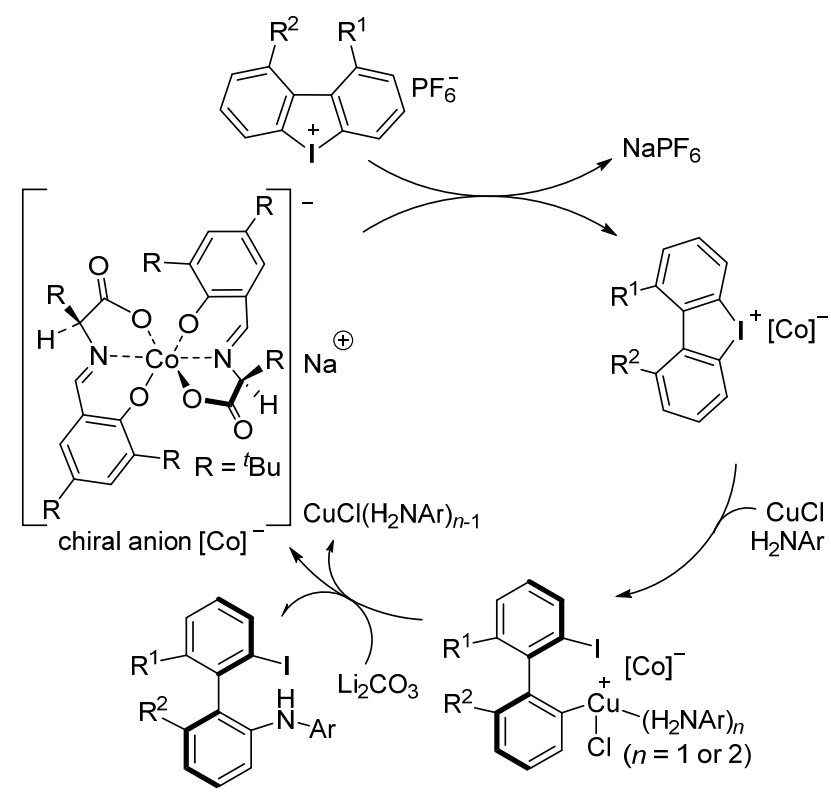

图式 4 可能的催化循环

Scheme 4 The proposed catalytic cycle
高碘盐形成紧密离子对, 这一物种相对于带有六氟磷酸 根的高碘盐, 具有更高的反应活性, 会优先与氯化亚铜 芳胺配合物发生氧化加成开环反应，随后经过还原消除 步骤得到轴手性化合物，同时释放铜催化剂.

总结，顾振华、俞杰等在铜催化环状芳基高碘盐的 开环反应中, 利用手性钴阴离子为添加剂, 成功解决了 先前大位阻芳胺为亲核试剂时的反应活性和立体选择 性不能得到有效控制的问题. 这一结果表明在金属催化 反应中，新型手性阴离子催化剂的开发和应用具有广阔 的前景, 有助于吸引研究者进一步尝试手性阴离子催化 剂在不对称反应中的应用.

\section{References}

[1] Lassaletta, J. M. Atropisomerism and Axial Chirality, World Scientific, New Jersey, 2019.

[2] Wencel-Delord, J.; Panossian, A.; Leroux, F. R.; Colobert, F. Chem. Soc. Rev. 2015, 44, 3418.

[3] Yoshimura, A.; Zhdankin, V. V. Chem. Rev. 2016, 116, 3328.

[4] (a) Zhao, K.; Duan, L.; Xu, S.; Jiang, J.; Fu, Y.; Gu, Z. Chem 2018 , 4, 599 .

(b) Xu, S.; Zhao, K.; Gu, Z. Adv. Synth. Catal. 2018, 360, 3877.

[5] Phipps, R. J.; Hamilton, G. L.; Toste, F. D. Nat. Chem. 2012, 4, 603.

[6] (a) Yu, J.; Jiang, H.-J.; Zhou, Y.; Luo, S.-W.; Gong, L.-Z. Angew. Chem., Int. Ed. 2015, 54, 11209.

(b) Jiang, H.-J.; Liu, K.; Yu, J.; Zhang, L.; Gong, L.-Z. Angew. Chem., Int. Ed. 2017, 56, 11931.

(c) Jiang, H.-J.; Zhong, X.-M.; Yu, J.; Zhang, Y.; Zhang, X.; Wu, Y.-D.; Gong, L.-Z. Angew. Chem., Int. Ed. 2019, 58, 1803.

[7] Zhang, X.; Zhao, K.; Li, N.; Yu, J.; Gong, L.-Z.; Gu, Z. Angew. Chem., Int. Ed. 2020, 59, 19899.

(Cheng, F.) 